EFI-02-51

hep-th/0211218

\title{
D-Branes on the Null-Brane
}

\author{
Kazumi Okuyama \\ Enrico Fermi Institute, University of Chicago \\ 5640 S. Ellis Ave., Chicago IL 60637, USA \\ kazumi@theory.uchicago.edu
}

\begin{abstract}
We study D-branes in the null-brane background. Using the covariant formalism of the worldsheet theory, we construct the boundary states describing D-branes on the nullbrane. From the cylinder amplitudes, we find that the D-branes with codimension zero or two have time-dependent effective tensions.
\end{abstract}

November 2002 


\section{Introduction}

It is important to study string theory in various curved backgrounds since our understanding of string theory is not background independent. In particular, it is interesting to study string theory in time-dependent backgrounds. Time-dependent orbifolds of flat space would be good toy models for more realistic cosmological backgrounds in string theory [1]21].

The null-brane [3:7] is an orbifold of $\mathbf{R}^{1,3}$ which has several good properties as a background of string theory: It is a smooth supersymmetric orbifold and does not contain closed time-like curves. It was shown that the gravitational backreaction due to the introduction of a single particle in this background is supressed when the number of noncompact dimensions is larger than five [18], and that the tree level string amplitude makes sence in this background [16, [17].

It is interesting to study the behavior of solitonic objects in time-dependent backgrounds. In this paper, we consider D-branes in the null-brane background and construct the boundary states describing them. After reviewing the geometry of the null-brane in section 1, in section 2 we consider the closed string theory on the null-brane. We find that the Virasoro operator in the twisted sector is related to the one in the untwisted sector by a spectral flow. Using the operator formalism, we rederive the torus amplitude obtained in [16, 17]. In section 3, we construct the boundary states for D3- and D2-branes, and compute the cylinder amplitudes. We find that the effective tension of the D3-brane is time-dependent. In section 4, we consider D1-brane and discuss its relation to the nullscissors configuration discussed recently in [22]. Section 5 is devoted to discussions. In appendix A, we consider the trace in the zero-mode part of a string, and in appendix B we discuss the T-dual of the null-scissors.

\section{Geometry of the Null-Brane}

The null-brane is an orbifold of a flat 4-dimensional Minkowski space $\mathbf{R}^{1,2} \times \mathbf{R}$ with the metric

$$
d s^{2}=\eta_{\mu \nu} d x^{\mu} d x^{\nu}+d z^{2}=-2 d x^{+} d x^{-}+d x^{2}+d z^{2} .
$$

The orbifold group $\Gamma \simeq \mathbf{Z}$ acts as a null-boost on $x^{\mu}(\mu=+, x,-)$ and shifts $z$ by a constant:

$$
X=\left(\begin{array}{c}
x^{+} \\
x \\
x^{-}
\end{array}\right) \mapsto e^{2 \pi \mathcal{J}} X, \quad z \mapsto z+2 \pi R .
$$


Above, the matrix $\mathcal{J}$ is the vector representation of the generator $J=\left(J^{0 x}+J^{1 x}\right) / \sqrt{2}$, and its explicit form is given by

$$
\mathcal{J}=\left(\begin{array}{lll}
0 & 0 & 0 \\
1 & 0 & 0 \\
0 & 1 & 0
\end{array}\right)
$$

The important property of $\mathcal{J}$ is that it is nilpotent and belongs to the Lie algebra $s o(1,2)$

$$
\mathcal{J}^{3}=0, \quad \mathcal{J}^{T}=-\eta \mathcal{J} \eta^{-1}
$$

where the superscript $T$ denotes the transpose of the matrix.

To describe the geometry of the orbifold $\left(\mathbf{R}^{1,2} \times \mathbf{R}\right) / \Gamma$, it is convenient to introduce a new set of coordinates $\left(y^{+}, y, y^{-}, u\right)$ defined as

$$
\left(\begin{array}{c}
x^{+} \\
x \\
x^{-}
\end{array}\right)=e^{y \mathcal{J}}\left(\begin{array}{c}
y^{+} \\
0 \\
y^{-}
\end{array}\right)=\left(\begin{array}{c}
y^{+} \\
y y^{+} \\
y^{-}+\frac{1}{2} y^{2} y^{+}
\end{array}\right), \quad z=u+R y
$$

In terms of these coordinates, the orbifold action (2.2) becomes simply $y \sim y+2 \pi$ with other coordinates kept invariant. The metric on the null-brane in these variables becomes

$$
d s^{2}=-2 d y^{+} d y^{-}+\left[R^{2}+\left(y^{+}\right)^{2}\right](d y+A)^{2}+\frac{\left(y^{+}\right)^{2}}{R^{2}+\left(y^{+}\right)^{2}} d u^{2}
$$

where $A$ is the connection of the $\mathbf{S}^{1}$ fiber with non-vanishing curvature

$$
A=\frac{R}{R^{2}+\left(y^{+}\right)^{2}} d u, \quad F=d A=-\frac{R y^{+}}{R^{2}+\left(y^{+}\right)^{2}} d y^{+} \wedge d u
$$

\section{Closed Strings on the Null-Brane}

\subsection{Mode Expansion in the Twisted Sector}

To construct the boundary states on the null-brane, let us begin with the mode expansion of worldsheet fields in the covariant formalism. First we focus on the $\mathbf{R}^{1,2}$ part of the target space whose worldsheet action is

$$
S=\frac{1}{4 \pi \alpha^{\prime}} \int d t \int_{0}^{2 \pi} d \sigma\left(\partial_{t} X^{\mu} \partial_{t} X_{\mu}-\partial_{\sigma} X^{\mu} \partial_{\sigma} X_{\mu}\right)=\frac{1}{\pi \alpha^{\prime}} \int d t \int_{0}^{2 \pi} d \sigma \partial_{+} X^{T} \eta \partial_{-} X
$$

where

$$
u^{ \pm}=t \pm \sigma, \quad \partial_{ \pm} \equiv \frac{\partial}{\partial u^{ \pm}}=\frac{1}{2}\left(\partial_{t} \pm \partial_{\sigma}\right)
$$


In the $w^{\text {th }}$ twisted sector, $X(t, \sigma)$ is periodic up to a $\Gamma$-action

$$
X(t, \sigma+2 \pi)=e^{2 \pi w \mathcal{J}} X(t, \sigma)
$$

The mode expansion of $X$ in this sector is given by 8

$$
X=i \sqrt{\frac{\alpha^{\prime}}{2}} \sum_{n \in \mathbf{Z}}\left(\frac{e^{-i(n+i w \mathcal{J}) u^{+}}}{n+i w \mathcal{J}} \alpha_{n}^{(w)}+\frac{e^{-i(n-i w \mathcal{J}) u^{-}}}{n-i w \mathcal{J}} \widetilde{\alpha}_{n}^{(w)}\right)
$$

and its conjugate momentum is

$$
\Pi=\frac{\partial_{t} X}{2 \pi \alpha^{\prime}}=\frac{1}{2 \pi \sqrt{2 \alpha^{\prime}}} \sum_{n \in \mathbf{Z}}\left(e^{-i(n+i w \mathcal{J}) u^{+}} \alpha_{n}^{(w)}+e^{-i(n-i w \mathcal{J}) u^{-}} \widetilde{\alpha}_{n}^{(w)}\right) .
$$

The zero modes are written as

$$
\alpha_{0}^{(w)}=\sqrt{\frac{\alpha^{\prime}}{2}}\left(p+\frac{w \mathcal{J}}{\alpha^{\prime}} x\right), \quad \widetilde{\alpha}_{0}^{(w)}=\sqrt{\frac{\alpha^{\prime}}{2}}\left(p-\frac{w \mathcal{J}}{\alpha^{\prime}} x\right),
$$

where $x^{\mu}$ and $p^{\mu}$ satisfy the canonical commutation relation $\left[x^{\mu}, p^{\nu}\right]=i \eta^{\mu \nu}$. At $t=0$, (3.4) and (3.5) reduce to

$$
\begin{aligned}
& X=e^{w \sigma \mathcal{J}}\left[x+i \sqrt{\frac{\alpha^{\prime}}{2}} \sum_{n \neq 0} \frac{e^{-i n \sigma}}{n+i w \mathcal{J}}\left(\alpha_{n}^{(w)}-\widetilde{\alpha}_{-n}^{(w)}\right)\right] \equiv e^{w \sigma \mathcal{J}} \widetilde{X} \\
& \Pi=\frac{1}{2 \pi} e^{w \sigma \mathcal{J}}\left[p+\frac{1}{\sqrt{2 \alpha^{\prime}}} \sum_{n \neq 0} e^{-i n \sigma}\left(\alpha_{n}^{(w)}+\widetilde{\alpha}_{-n}^{(w)}\right)\right] \equiv e^{w \sigma \mathcal{J}} \widetilde{\Pi} .
\end{aligned}
$$

From the relation $\left[X^{\mu}(\sigma), \Pi_{\nu}\left(\sigma^{\prime}\right)\right]=i \delta_{\nu}^{\mu} \delta\left(\sigma, \sigma^{\prime}\right)$, the commutation relations of the non-zero modes are found to be

$$
\begin{aligned}
& {\left[\alpha_{n}^{(w) \mu}, \alpha_{m}^{(w) \nu}\right]=\left(n \eta^{-1}+i w \mathcal{J} \eta^{-1}\right)^{\mu \nu} \delta_{n+m, 0}} \\
& {\left[\widetilde{\alpha}_{n}^{(w) \mu}, \widetilde{\alpha}_{m}^{(w) \nu}\right]=\left(n \eta^{-1}-i w \mathcal{J} \eta^{-1}\right)^{\mu \nu} \delta_{n+m, 0}}
\end{aligned}
$$

More explicitly, the non-vanishing commutators for the left movers are

$$
\left[\alpha_{n}^{(w) x}, \alpha_{m}^{(w) x}\right]=n \delta_{n+m, 0}, \quad\left[\alpha_{n}^{(w)+}, \alpha_{m}^{(w)-}\right]=-n \delta_{n+m, 0}, \quad\left[\alpha_{n}^{(w)-}, \alpha_{m}^{(w) x}\right]=i w \delta_{n+m, 0}
$$

Next consider the field $Z(t, \sigma)$ in the twisted sector $Z(t, \sigma+2 \pi)=Z(t, \sigma)+2 \pi w R$. The mode expansion of $Z$ is the familiar winding configuration on a circle:

$$
Z=z+\sqrt{\frac{\alpha^{\prime}}{2}}\left(\alpha_{0}^{(w) z} u^{+}+\widetilde{\alpha}_{0}^{(w) z} u^{-}\right)+i \sqrt{\frac{\alpha^{\prime}}{2}} \sum_{n \neq 0} \frac{1}{n}\left(e^{-i n u^{+}} \alpha_{n}^{z}+e^{-i n u^{-}} \widetilde{\alpha}_{n}^{z}\right)
$$


where $\left[\alpha_{n}^{z}, \alpha_{m}^{z}\right]=n \delta_{n+m, 0}$ and

$$
\alpha_{0}^{(w) z}=\sqrt{\frac{\alpha^{\prime}}{2}} p_{z}+\frac{w R}{\sqrt{2 \alpha^{\prime}}}, \quad \widetilde{\alpha}_{0}^{(w) z}=\sqrt{\frac{\alpha^{\prime}}{2}} p_{z}-\frac{w R}{\sqrt{2 \alpha^{\prime}}}
$$

\subsection{Spectral Flow}

As pointed out in [8], the commutation relation (3.8) leads to an exchange algebra of the currents $\partial_{ \pm} X^{\mu}$. Therefore, one might think that the quantization of the twisted sector would be quite complicated. However, by a simple change of variables the relation (3.8) reduces to the ordinary commutation relation of oscillators. To see this, we introduce the following invertible matrix $\mathcal{S}_{\beta}$

$$
\mathcal{S}_{\beta}=1+\frac{i}{2} \beta \mathcal{J}+\frac{1}{8} \beta^{2} \mathcal{J}^{2}, \quad\left(\mathcal{S}_{\beta}\right)^{-1}=1-\frac{i}{2} \beta \mathcal{J}-\frac{3}{8} \beta^{2} \mathcal{J}^{2}
$$

which satisfies

$$
\left(\mathcal{S}_{\beta}\right)^{2}=1+i \beta \mathcal{J}, \quad\left(\mathcal{S}_{\beta}\right)^{T}=\eta \mathcal{S}_{-\beta} \eta^{-1}
$$

In terms of this, the commutation relation (3.8) is rewritten as

$$
\begin{aligned}
& {\left[\alpha_{n}^{(w) \mu}, \alpha_{m}^{(w) \nu}\right]=n \delta_{n+m, 0}\left(\mathcal{S}_{\frac{w}{n}} \eta^{-1} \mathcal{S}_{\frac{w}{m}}^{T}\right)^{\mu \nu},} \\
& {\left[\widetilde{\alpha}_{n}^{(w) \mu}, \widetilde{\alpha}_{m}^{(w) \nu}\right]=n \delta_{n+m, 0}\left(\mathcal{S}_{-\frac{w}{n}} \eta^{-1} \mathcal{S}_{-\frac{w}{m}}^{T}\right)^{\mu \nu} .}
\end{aligned}
$$

In other words, $\mathcal{S}_{ \pm \frac{w}{n}}$ are the square root of the matrices in the right-hand sides of (3.8). Therefore, the oscillators in the twisted sector and the ordinary untwisted oscillators are related by the following "spectral flow"

$$
\alpha_{n}^{(w)}=\mathcal{S}_{\frac{w}{n}} \alpha_{n}^{(0)}, \quad \widetilde{\alpha}_{n}^{(w)}=\mathcal{S}_{-\frac{w}{n}} \widetilde{\alpha}_{n}^{(0)}
$$

Namely, by taking a linear combination of the oscillators in the twisted sector, we can construct $\alpha_{n}^{(0)}$ and $\widetilde{\alpha}_{n}^{(0)}$ obeying the ordinary relation

$$
\left[\alpha_{n}^{(0) \mu}, \alpha_{m}^{(0) \nu}\right]=\left[\widetilde{\alpha}_{n}^{(0) \mu}, \widetilde{\alpha}_{m}^{(0) \nu}\right]=n \delta_{n+m, 0} \eta^{\mu \nu}
$$

The oscillator vacuum in the $w^{\text {th }}$ twisted sector is naturally defined as

$$
\alpha_{n}^{(w) \mu}|0\rangle_{w}=\alpha_{n}^{(0) \mu}|0\rangle_{w}=0 \quad(n>0) .
$$




\subsection{Symmetry Generators}

In this subsection, we construct the generator of the null-boost and the Virasoro operator $L_{0}$ in the twisted sector.

Let us first consider the null-boost generator $\widehat{J}$ :

$$
\widehat{J}=\int_{0}^{2 \pi} d \sigma\left(X^{+} \Pi^{x}-X^{x} \Pi^{+}\right)=-\int_{0}^{2 \pi} d \sigma X^{T} \eta \mathcal{J} \Pi=-\int_{0}^{2 \pi} d \sigma \widetilde{X}^{T} \eta \mathcal{J} \widetilde{\Pi}
$$

where the variables $\widetilde{X}$ and $\widetilde{\Pi}$ are defined in (3.7). In the last equality we used the fact that $e^{w \sigma \mathcal{J}}$ preserves $\eta$. Now $\widehat{J}$ is written in terms of the modes as

$$
\widehat{J}=J_{0}+E+\widetilde{E},
$$

where

$$
\begin{aligned}
& J_{0}=-x^{T} \eta \mathcal{J} p=x^{+} p^{x}-x p^{+} \\
& E=\frac{i}{2} \sum_{n \neq 0} \frac{1}{n} \alpha_{-n}^{(0) T} \eta \mathcal{J} \alpha_{n}^{(0)}=i \sum_{n \neq 0} \frac{1}{n} \alpha_{n}^{(0)+} \alpha_{-n}^{(0) x}, \quad \widetilde{E}=i \sum_{n \neq 0} \frac{1}{n} \widetilde{\alpha}_{n}^{(0)+} \widetilde{\alpha}_{-n}^{(0) x} .
\end{aligned}
$$

Here we wrote $E$ and $\widetilde{E}$ in terms of the untwisted oscillators.

Next consider the stress tensor in the twisted sector. The $\mathbf{R}^{1,2} \times \mathbf{R}$ part of the stress tensor is given by

$$
T=\frac{1}{\alpha^{\prime}} \partial_{+} X^{\mu} \partial_{+} X_{\mu}+\frac{1}{\alpha^{\prime}}\left(\partial_{+} Z\right)^{2} \equiv \sum_{n \in \mathbf{Z}} e^{-i n \sigma} L_{n}^{(w)} .
$$

By substituting the mode expansion, $L_{0}$ in the $w^{\text {th }}$ twisted sector is found to be

$$
L_{0}^{(w)}=\frac{1}{2} \sum_{n \in \mathbf{Z}} \alpha_{n}^{(w) \mu} \alpha_{-n, \mu}^{(w)}+\frac{1}{2} \sum_{n \in \mathbf{Z}} \alpha_{n}^{z} \alpha_{-n}^{z} .
$$

We can rewrite $L_{0}^{(w)}$ in terms of the untwisted oscillator using the spectral flow (3.15):

$$
\begin{aligned}
& L_{0}^{(w)}=\frac{\alpha^{\prime}}{4}\left(p^{\mu} p_{\mu}+p_{z}^{2}\right)+N^{(0)}+\frac{w}{2}\left(J_{0}+p_{z} R+2 E\right)+\frac{\alpha^{\prime}}{4}\left(\frac{2 \pi w R_{+}}{2 \pi \alpha^{\prime}}\right)^{2} \\
& \widetilde{L}_{0}^{(w)}=\frac{\alpha^{\prime}}{4}\left(p^{\mu} p_{\mu}+p_{z}^{2}\right)+\widetilde{N}^{(0)}-\frac{w}{2}\left(J_{0}+p_{z} R+2 \widetilde{E}\right)+\frac{\alpha^{\prime}}{4}\left(\frac{2 \pi w R_{+}}{2 \pi \alpha^{\prime}}\right)^{2}
\end{aligned}
$$

where $N^{(0)}$ and $\tilde{N}^{(0)}$ are the number operators of the untwisted oscillators, and $R_{+}$is defined as

$$
R_{+}=\sqrt{\left(x^{+}\right)^{2}+R^{2}}
$$

The last term in (3.23) can be understood as the (mass) ${ }^{2}$ of the string winding $w$-times around the $\mathbf{S}^{1}$ fiber of the null-brane with radius $R_{+}$.

We end this subsection with a comment on the non-zero modes $L_{n}^{(w)}$ of the Virasoro generator. $L_{n}^{(w)}$ does not have a good transformation property under the "spectral flow" (3.15), since the integrand of $\widehat{J}$ is not a conformal current. 


\subsection{Torus Amplitute}

As a consistency check of the result in the previous subsection, we compute the 1-loop partition sum

$$
Z=\sum_{k, w \in \mathbf{Z}} \operatorname{Tr}_{w} e^{-2 \pi i k\left(\widehat{J}+p_{z} R\right)} q_{-}^{L_{0}-\frac{c}{24}} q_{+}^{\widetilde{L}_{0}-\frac{c}{24}}
$$

which was computed by the path integral formalism in [8, 16, 17]. Here we defined $q_{ \pm}=$ $e^{\mp 2 \pi i \tau_{ \pm}}$and $\tau_{ \pm}=\tau_{1} \pm \tau_{2} . \operatorname{Tr}_{w}$ means the trace in the $w^{\text {th }}$ twisted sector, and the sum over $k$ imposes the invariance under the orbifold projection. As emphasized in [8], we have to take the worldsheet metric to be Lorentzian since $L_{0}$ is unbounded from below.

Let us first consider the zero-mode part. Because the trace is independent of the choice of basis, we can compute the trace in the plane wave basis. In this basis, we can argue that the trace can be replaced by a classical phase space integral which is a Gaussian integral (see appendix A). I Since $p^{-}$appears linearly in $L_{0}^{(w)}$ in the form $p^{+} p^{-}$, the integral over $p^{-}$gives the delta-function $\delta\left(p^{+}\right)$. Therefore, only $p^{+}=0$ states contribute to the partition sum. In this computation, it is important to take $\tau_{ \pm}$to be real. After performing the Gaussian integral over $p_{x}$ and $p_{z}$, we get the factor

$$
\sum_{k, w \in \mathbf{Z}} \exp \left(i \frac{\pi R_{+}^{2}}{\alpha^{\prime} \tau_{2}}\left(w \tau_{+}-k\right)\left(w \tau_{-}-k\right)\right)
$$

which agrees with the contribution of the classical action in the path integral formalism.

For the non-zero modes, one can show that the trace is independent of $w$ by the same reason as the path integral computation. Since $E$ in (3.20) commutes with $N^{(0)}$, we can focus on a fixed level. By noting that the number basis of oscillators

$$
\left|N_{m}^{+}, N_{m}^{x}, N_{m}^{-}\right\rangle=\frac{1}{\sqrt{N_{m}^{+} ! N_{m}^{x} ! N_{m}^{-} !}}\left(\frac{\alpha_{-m}^{(0)+}}{\sqrt{m}}\right)^{N_{m}^{+}}\left(\frac{\alpha_{-m}^{(0) x}}{\sqrt{m}}\right)^{N_{m}^{x}}\left(\frac{\alpha_{-m}^{(0)-}}{\sqrt{m}}\right)^{N_{m}^{-}}|0\rangle
$$

1 We can also compute the trace in the $J$-eigenbasis. In this case, we have to redefine the wavefunction $\psi_{\mathrm{LMS}}$ in 16 as

$$
\psi_{\text {new }}=\exp \left(-i \frac{J^{2}}{2 p^{+} x^{+}}\right) \psi_{\mathrm{LMS}}, \quad\left[p^{2}+m^{2}-\frac{J^{2}}{\left(x^{+}\right)^{2}}\right] \psi_{\text {new }}=0,
$$

since $\psi_{\text {LMS }}$ is singular in the limit $p^{+} \rightarrow 0$. Note that $J^{2} /\left(x^{+}\right)^{2}$ is the rotation energy around the $\mathbf{S}^{1}$ fiber. 
transforms under the null-boost as

$$
\begin{aligned}
& e^{i v E}\left|N_{m}^{+}, N_{m}^{x}, N_{m}^{-}\right\rangle \\
= & \frac{1}{\sqrt{N_{m}^{+} ! N_{m}^{x} ! N_{m}^{-} !}}\left(\frac{\alpha_{-m}^{(0)+}}{\sqrt{m}}\right)^{N_{m}^{+}}\left(\frac{\alpha_{-m}^{(0) x}+v \alpha_{-m}^{(0)+}}{\sqrt{m}}\right)^{N_{m}^{x}}\left(\frac{\alpha_{-m}^{(0)-}+v \alpha_{-m}^{(0) x}+\frac{v^{2}}{2} \alpha_{-m}^{(0)+}}{\sqrt{m}}\right)^{N_{m}^{-}}|0\rangle,
\end{aligned}
$$

and using the definition of the conjugate $\left(\alpha_{n}^{(0) x}\right)^{\dagger}=\alpha_{-n}^{(0) x},\left(\alpha_{n}^{(0) \pm}\right)^{\dagger}=-\alpha_{-n}^{(0) \mp}$, one can easily see that the matrix element of $q^{N^{(0)}} e^{i v E}$ is $v$-independent:

$$
\left\langle N_{m}^{+}, N_{m}^{x}, N_{m}^{-}\left|q^{N^{(0)}} e^{i v E}\right| N_{m}^{+}, N_{m}^{x}, N_{m}^{-}\right\rangle=\left\langle N_{m}^{+}, N_{m}^{x}, N_{m}^{-}\left|q^{N^{(0)}}\right| N_{m}^{+}, N_{m}^{x}, N_{m}^{-}\right\rangle
$$

This independence of the twist $w$ in the non-zero mode part is related to the fact that in the Fock space there is no eigenstate of $E$ with non-zero eigenvalue, since $\mathcal{J}$ is nilpotent in all finite dimensional representations. Only $J_{0}$ is diagonalizable on the infinite dimensional space of functions.

Finally, we find that the operator formalism (3.25) gives the same result as the path integral formalism:

$$
Z=\frac{Z^{\text {ghost }} Z^{\perp}}{2 \pi i \tau_{2}^{2} \eta\left(q_{-}\right)^{4} \eta\left(q_{+}\right)^{4}} \int \frac{d^{3} x d z}{\left(2 \pi \sqrt{\alpha^{\prime}}\right)^{4}} \sum_{k, w \in \mathbf{Z}} \exp \left(i \frac{\pi R_{+}^{2}}{\alpha^{\prime} \tau_{2}}\left(w \tau_{+}-k\right)\left(w \tau_{-}-k\right)\right) .
$$

\subsection{Superstring in the NSR Formalism}

In this subsection, we briefly comment on the extension to the superstring in the NSR formalism. In the $w^{\text {th }}$ twisted sector, the mode expansion of the fermions in the $\mathbf{R}^{1,2}$ part is given by

$$
\psi=\sum_{r \in \mathbf{Z}+\nu} e^{-i(r+i w \mathcal{J}) u^{+}} \psi_{r}, \quad \widetilde{\psi}=\sum_{r \in \mathbf{Z}+\nu} e^{-i(r-i w \mathcal{J}) u^{-}} \widetilde{\psi}_{r}
$$

where $\nu=\frac{1}{2}$ for the NS sector and $\nu=0$ for the $\mathrm{R}$ sector. The commutation relation is the same as the untwisted sector:

$$
\left\{\psi_{r}^{\mu}, \psi_{s}^{\nu}\right\}=\left\{\widetilde{\psi}_{r}^{\mu}, \widetilde{\psi}_{s}^{\nu}\right\}=\eta^{\mu \nu} \delta_{r+s, 0}
$$

The $L_{0}$ in the twisted sector computed from the stress tensor

$$
T=\frac{i}{2} \psi^{T} \eta \partial_{+} \psi, \quad \bar{T}=\frac{i}{2} \widetilde{\psi}^{T} \eta \partial_{-} \widetilde{\psi}
$$


has a similar "spectral flowed" form as that of the bosonic counterpart:

$$
L_{0}^{(w)}=N_{\psi}+w E_{\psi}, \quad \widetilde{L}_{0}^{(w)}=\widetilde{N}_{\psi}-w \widetilde{E}_{\psi}
$$

where $N_{\psi}$ and $E_{\psi}$ are the number operator and the null-boost generator, respectively

$$
N_{\psi}=\frac{1}{2} \sum_{r \in \mathbf{Z}+\nu} r \psi_{-r}^{T} \eta \psi_{r}, \quad E_{\psi}=\frac{i}{2} \sum_{r \in \mathbf{Z}+\nu} \psi_{-r}^{T} \eta \mathcal{J} \psi_{r}
$$

$\widetilde{N}_{\psi}$ and $\widetilde{E}_{\psi}$ are obtained by putting a tilde on $\psi$ in the above expressions. The mode expansion and the commutation relation of $\psi^{z}$ are the same as the untwisted sector.

The torus amplitude in the superstring case is obtained by the same formula (3.31) by including the contribution of fermions in $Z_{\perp}[8]$. The important point is that the partition function of fermions is independent of the twist $w$

$$
Z_{\psi}^{(w)}=Z_{\psi}^{(0)}
$$

by the same reason as the non-zero modes of the bosonic part.

\section{Boundary States on the Null-Brane}

In this section, we construct the boundary states describing BPS D3- and D2-branes on the null-brane background which are extended along the $X^{\mu}$ directions. The supersymmetry of branes in the null-brane geometry was considered in [23,24]. It turns out to be easy to construct these states since the boundary condition is invariant under the null-boost.

\subsection{D3-Branes}

Let us first construct the D3-brane which wraps the whole null-brane. On the covering space $\mathbf{R}^{1,2} \times \mathbf{R}, X^{\mu}$ and $Z$ satisfy the Neumann boundary condition.

$$
\partial_{t} X^{\mu}(t=0)|D 3\rangle=\partial_{t} Z(t=0)|D 3\rangle=0
$$

In the $w^{\text {th }}$ twisted sector the boundary conditions for modes read

$$
p^{\mu}|D 3\rangle_{w}=p_{z}|D 3\rangle_{w}=0, \quad\left(\alpha_{n}^{(w) \mu}+\widetilde{\alpha}_{-n}^{(w) \mu}\right)|D 3\rangle_{w}=\left(\alpha_{n}^{z}+\widetilde{\alpha}_{-n}^{z}\right)|D 3\rangle_{w}=0
$$


By the spectral flow, the condition for $\alpha_{n}^{(w)}$ and $\widetilde{\alpha}_{n}^{(w)}$ is rewritten as

$$
\left(\alpha_{n}^{(0) \mu}+\widetilde{\alpha}_{-n}^{(0) \mu}\right)|D 3\rangle_{w}=0
$$

Since this is the ordinary Neumann boundary condition, we can easily write down the boundary state in the twisted sector:

$$
\begin{aligned}
|D 3\rangle_{w} & =\exp \left(\sum_{n>0}-\frac{1}{n} \alpha_{-n}^{(0) T} \eta \widetilde{\alpha}_{-n}^{(0)}-\frac{1}{n} \alpha_{-n}^{z} \widetilde{\alpha}_{-n}^{z}\right)\left|p^{\mu}=p_{z}=0\right\rangle_{w} \\
& =\exp \left(\sum_{n>0}-\alpha_{-n}^{(w) T} \eta \frac{1}{n+i w \mathcal{J}} \widetilde{\alpha}_{-n}^{(w)}-\frac{1}{n} \alpha_{-n}^{z} \widetilde{\alpha}_{-n}^{z}\right)\left|p^{\mu}=p_{z}=0\right\rangle_{w} .
\end{aligned}
$$

This state is invariant under the orbifold action

$$
e^{2 \pi i\left(\widehat{J}+p_{z} R\right)}|D 3\rangle_{w}=0
$$

and hence becomes a well-defined state on the orbifold. This invariance automatically follows from the boundary conditions (4.2).

For the fermions, the boundary state in the twisted sector is the same as the untwisted sector

$$
|D 3\rangle_{\psi, w}=|D 3\rangle_{\psi, w=0}
$$

Now the complete boundary state of D3-brane is obtained by summing all the twisted sector states

$$
\left|D 3_{\theta}\right\rangle=\sum_{w \in \mathbf{Z}} e^{i w \theta}|D 3\rangle_{w}
$$

Here we introduced a Wilson line $\theta$.

To compute the potential between two D3-branes, let us consider the cylinder amplitude

$$
\mathcal{A}_{\text {closed }}=\int_{0}^{\infty} \frac{d s}{s}\left\langle D 3_{\theta}\left|e^{-\pi s\left(L_{0}+\widetilde{L}_{0}-\frac{c}{12}\right)}\right| D 3_{\theta^{\prime}}\right\rangle=\int_{0}^{\infty} \frac{d s}{s} \mathcal{A}_{0}(s) \mathcal{A}^{\prime}(s) .
$$

Here we factored out the contribution of the zero-mode of $X^{\mu}$ and $Z$ as $\mathcal{A}_{0}(s)$. $\mathcal{A}^{\prime}(s)$ is the contribution from the transverse coordinates, the fermions, and the non-zero modes of $X^{\mu}$ and $Z$. Plugging the expression of $L_{0}$ and $\widetilde{L}_{0}(3.23)$ into this amplitude $\mathcal{A}_{\text {closed }}$, the contribution of the zero-mode turns out to be

$$
\mathcal{A}_{0}(s)=\int d^{3} x d z \sum_{w \in \mathbf{Z}} \exp \left[-\pi s \frac{w^{2} R_{+}^{2}}{2 \alpha^{\prime}}-i w\left(\theta-\theta^{\prime}\right)\right] .
$$


One can show that the rest of the contribution $\mathcal{A}^{\prime}(s)$ is independent of the twist $w$ in the same way as the computation of the torus amplitude. Especially, $\mathcal{A}^{\prime}(s)$ vanishes in the Type IIB string if we take the boundary state of fermions $|D 3\rangle_{\psi, w}=|D 3\rangle_{\psi, w=0}$ as that of the BPS D3-brane on the flat space. Therefore, the effect of the orbifolding is contained entirely in $\mathcal{A}_{0}(s)$. By performing the Poisson resummation

$$
\sum_{w \in \mathbf{Z}} \exp \left[-\pi s w^{2} \frac{R_{+}^{2}}{2 \alpha^{\prime}}-i w\left(\theta-\theta^{\prime}\right)\right]=\frac{\sqrt{2 \alpha^{\prime}}}{\sqrt{s} R_{+}} \sum_{n \in \mathbf{Z}} \exp \left[-\frac{2 \pi \alpha^{\prime}}{s R_{+}^{2}}\left(n+\frac{\theta-\theta^{\prime}}{2 \pi}\right)^{2}\right],
$$

we can see that the effective tension of the D3-brane near $x^{+}=0$ behaves as

$$
\mathcal{T}_{D 3} \sim\left[\left(x^{+}\right)^{2}+R^{2}\right]^{-\frac{1}{4}} .
$$

This can be thought of as an open string analog of the time-dependent cosmological constant obtained from the torus amplitude of the closed string [8,16,17]. Strictly speaking, this is not the tension of the brane defined as the strength of the coupling to the graviton. The time dependence (4.11) comes from the exchange of twisted closed string states.

We can also compute this cylinder amplitude as a 1-loop amplitude of the open string. The open string connecting two D3-branes satisfies the boundary condition

$$
\partial_{\sigma} X^{\mu}=\partial_{\sigma} Z=\partial_{t} X_{\perp}=0 \text { at } \sigma=0, \pi
$$

and the Virasoro generator has the standard form $L_{0}=\alpha^{\prime}\left(p^{\mu} p_{\mu}+p_{z}^{2}\right)+N$. In the open string channel, the cylinder amplitude (4.8) is written as

$$
\mathcal{A}_{\mathrm{open}}=\int_{0}^{\infty} \frac{d t}{t} \sum_{w \in \mathbf{Z}} e^{i w\left(\theta^{\prime}-\theta\right)} \operatorname{Tr}\left[e^{2 \pi i w\left(\widehat{J}+p_{z} R\right)} e^{-2 \pi t\left(L_{0}-\frac{c}{24}\right)}\right]=\int_{0}^{\infty} \frac{d t}{t} \widetilde{\mathcal{A}}_{0}(t) \widetilde{\mathcal{A}}^{\prime}(t)
$$

In this computation, we have to perform the inverse Wick rotation to make the Schwinger parameter $t$ pure imaginary. As above, $\widetilde{\mathcal{A}}_{0}(t)$ is the contribution from the zero-mode of $X^{\mu}$ and $Z$, and the rest of the contribution is denoted as $\widetilde{\mathcal{A}}^{\prime}(t)$. By performing the Gaussian integral over $p^{\mu}$ and $p_{z}, \widetilde{\mathcal{A}}_{0}(t)$ is calculated as

$$
\widetilde{\mathcal{A}}_{0}(t)=\frac{i}{2 \pi t^{2}} \int \frac{d^{3} x d z}{\left(2 \pi \sqrt{2 \alpha^{\prime}}\right)^{4}} \sum_{w \in \mathbf{Z}} \exp \left(-\frac{\pi}{t} \frac{w^{2} R_{+}^{2}}{2 \alpha^{\prime}}-i w\left(\theta-\theta^{\prime}\right)\right),
$$

and $\widetilde{\mathcal{A}^{\prime}}(t)$ is independent of $w$ by the same reason as above. Under the modular transformation $s=1 / t, \mathcal{A}_{\text {open }}$ agrees with $\mathcal{A}_{\text {closed }}$ up to a normalization constant. 


\subsection{D2-Branes}

Next we consider the D2-brane extending along $X^{\mu}$ and localized along $Z$ in the covering space. The boundary condition is given by

$$
\partial_{t} X^{\mu}(t=0)|D 2\rangle=\partial_{\sigma} Z(t=0)|D 2\rangle=0 .
$$

Due to the Dirichlet condition for $Z$, the D2-brane does not couple to the twisted sector. Therefore, the boundary state $|D 2\rangle$ is the same as that on the flat space:

$$
|D 2\rangle=\sum_{k \in \mathbf{Z}} \exp \left(\sum_{n>0}-\frac{1}{n} \alpha_{-n}^{T} \eta \widetilde{\alpha}_{-n}+\frac{1}{n} \alpha_{-n}^{z} \widetilde{\alpha}_{-n}^{z}\right) e^{-i z \frac{k}{R}}\left|p^{\mu}=0, p_{z}=\frac{k}{R}\right\rangle_{w=0} .
$$

This state is invariant under the orbifold action. The tension of this brane is timeindependent. It is also easy to construct the S-brane localized in all $X^{\mu}$ directions since the boundary condition is null-boost invariant.

\section{D1-Branes and Null Scissors}

D1-branes on the null-brane are also BPS states when oriented appropriately on the null-brane. One of the BPS configuration of D1-brane on the null-brane is represented by a D1-brane extending along the $x^{ \pm}$directions on the covering space and its infinitely many images under the orbifold group. The configuration of the original D1-brane and one of its image brane is called "null-scissors" [22]. When the original brane on the covering space is sitting at $x=a, z=b$, the corresponding brane on the null-brane is described by a curve

$$
y=\frac{a}{y^{+}}, \quad u=b-\frac{a R}{y^{+}} .
$$

The divergence of $y$ near $y^{+}=x^{+}=0$ merely reflects the coordinate singularity at $x^{+}=0$. The phase shift of the $\mathbf{S}^{1}$ fiber is finite along the curve (5.1)

$$
\Delta \phi=\int_{x^{+}=-\infty}^{x^{+}=\infty}(d y+A)=\frac{\pi a}{R} .
$$

In the case of the parabolic orbifold $R=0$, this phase shift diverges and the D1-brane winds around the $\mathbf{S}^{1}$ fiber infinitely many times near $x^{+}=0$. When $R=0$, a D1-brane with $a=0$ and another brane with $a \neq 0$ intersect at $x^{+}=a / 2 \pi k, k \in \mathbf{Z}$. These intersection points accumulate on $x^{+}=0$. 


\subsection{Closed String Description}

We can construct the boundary state of D1-brane described above as

$$
|D 1 ; a, b\rangle=\sum_{k \in \mathbf{Z}} e^{2 \pi i k\left(\widehat{J}+p_{z} R\right)}|\widehat{D 1} ; a, b\rangle
$$

where $|\widehat{D 1} ; a, b\rangle$ is the boundary state on the covering space

$|\widehat{D 1} ; a, b\rangle=\exp \left[\sum_{n>0} \frac{1}{n}\left(\alpha_{-n}^{+} \widetilde{\alpha}_{-n}^{-}+\alpha_{-n}^{-} \widetilde{\alpha}_{-n}^{+}+\alpha_{-n}^{x} \widetilde{\alpha}_{-n}^{x}+\alpha_{-n}^{z} \widetilde{\alpha}_{-n}^{z}\right)\right]\left|p^{ \pm}=0, x=a, z=b\right\rangle$.

The summation over $k$ corresponds to putting infinitely many image branes under the orbifold action. The state (5.3) is invariant under the orbifold action by construction.

The cylinder amplitude between $|D 1 ; a, b\rangle$ and $|D 1 ; 0,0\rangle$ is written as

$$
\mathcal{A}_{\text {closed }}=\int_{0}^{\infty} \frac{d s}{s} \sum_{k \in \mathbf{Z}}\left\langle\widehat{D 1} ; 0,0\left|e^{2 \pi i k\left(\widehat{J}+p_{z} R\right)} e^{-\pi s\left(L_{0}+\widetilde{L}_{0}-\frac{c}{12}\right)}\right| \widehat{D 1} ; a, b\right\rangle .
$$

The contribution from the non-zero modes is $k$-independent as in the previous section. The zero-mode contribution is proportional to

$$
\int d x^{+} d x^{-} \sum_{k \in \mathbf{Z}} \exp \left[-\frac{\left(2 \pi k x^{+}-a\right)^{2}}{2 \pi s \alpha^{\prime}}-\frac{(2 \pi k R-b)^{2}}{2 \pi s \alpha^{\prime}}\right] \text {. }
$$

By performing a Poisson resummation, we can see that the effective tension becomes time-

dependent: $\mathcal{T}_{D 1} \sim\left[\left(x^{+}\right)^{2}+R^{2}\right]^{-\frac{1}{4}}$. This time dependence comes from the interactions between infinitely many image branes on the covering space. In the next subsection, we will see that the exponent in (5.6) can be interpreted as the length of the open string.

\subsection{Open String Description}

The cylinder amplitude (5.5) can be also computed as an open string 1-loop. For a fixed $k$, the open string in question is stretched between two D1-branes in the null-scissors configuration on the covering space. The boundary condition for this open string is

$$
\begin{aligned}
& \sigma=0: \quad \partial_{\sigma} X^{+}=0, \quad X^{x}=a, \quad \partial_{\sigma} X^{-}=0, \quad Z=b \\
& \sigma=\pi: \quad \partial_{\sigma} X^{+}=0, \quad X^{x}-2 \pi k X^{+}=0, \quad \partial_{\sigma}\left(X^{-}-2 \pi k X^{x}\right)=0, \quad Z=2 \pi k R .
\end{aligned}
$$


The mode expansion of $Z$ satisfying this boundary condition is given by

$$
Z=b+k\left(R-\frac{b}{2 \pi k}\right)\left(u^{+}-u^{-}\right)+i \sqrt{\frac{\alpha^{\prime}}{2}} \sum_{n \neq 0} \frac{1}{n}\left(e^{-i n u^{+}}-e^{-i n u^{-}}\right) \alpha_{n} .
$$

To find the mode expansion of $X^{\mu}$, it is convenient to perform a T-duality along $X^{x}$ direction [22]. The boundary condition in the T-dual picture is given by

$$
\left.\partial_{\sigma} Y\right|_{\sigma=0}=0, \quad \partial_{\sigma} Y-\left.\pi v \mathcal{J} \partial_{t} Y\right|_{\sigma=\pi}=0
$$

where $v=2 k$, and the mode expansion is

$$
Y=x+i \sqrt{\frac{\alpha^{\prime}}{2}} \sum_{n \in \mathbf{Z}} \frac{1}{n+i v \mathcal{J}}\left(e^{-i(n+i v \mathcal{J}) u^{+}}+e^{-i(n+i v \mathcal{J}) u^{-}}-2 \delta_{n, 0}\right) \alpha_{n} .
$$

Taking the T-dual again, the mode expansion of the original coordinate is obtained as

$$
\begin{aligned}
& X^{+}=x^{+}+\sqrt{\frac{\alpha^{\prime}}{2}}\left(u^{+}+u^{-}\right) \alpha_{0}^{+}+i \sqrt{\frac{\alpha^{\prime}}{2}} \sum_{n \neq 0} \frac{1}{n}\left(e^{-i n u^{+}}+e^{-i n u^{-}}\right) \alpha_{n}^{+} \\
& X^{x}=a+\sqrt{\frac{\alpha^{\prime}}{2}}\left[u^{+} \alpha_{0}^{x}+\frac{v}{2}\left(u^{+}\right)^{2} \alpha_{0}^{+}+i \sum_{n \neq 0} \frac{e^{-i n u^{+}}}{n}\left(\alpha_{n}^{x}+v\left(u^{+}-\frac{i}{n}\right) \alpha_{n}^{+}\right)\right]-\left(u^{+} \leftrightarrow u^{-}\right) \\
& X^{-}=x^{-}+\sqrt{\frac{\alpha^{\prime}}{2}}\left[u^{+} \alpha_{0}^{-}+\frac{v}{2}\left(u^{+}\right)^{2} \alpha_{0}^{x}+\frac{v^{3}}{6}\left(u^{+}\right)^{3} \alpha_{0}^{+}\right] \\
& +i \sqrt{\frac{\alpha^{\prime}}{2}} \sum_{n \neq 0} \frac{e^{-i n u+}}{n}\left[\alpha_{n}^{-}+v\left(u^{+}-\frac{i}{n}\right) \alpha_{n}^{x}+v^{2}\left(\frac{\left(u^{+}\right)^{2}}{2}-\frac{i u^{+}}{n}-\frac{1}{n^{2}}\right) \alpha_{n}^{+}\right]+\left(u^{+} \leftrightarrow u^{-}\right) .
\end{aligned}
$$

From the canonical commutation relation $\left[X^{\mu}(\sigma), \partial_{t} X^{\nu}\left(\sigma^{\prime}\right)\right]=2 \pi i \alpha^{\prime} \eta^{\mu \nu} \delta\left(\sigma, \sigma^{\prime}\right)$, the commutation relation of the non-zero modes is found to be

$$
\left[\alpha_{n}^{\mu}, \alpha_{m}^{\nu}\right]=\left(n \eta^{-1}+i v \mathcal{J} \eta^{-1}\right)^{\mu \nu} \delta_{n+m, 0}
$$

The zero mode satisfying the boundary condition (5.7) is given by

$$
\alpha_{0}^{+}=\sqrt{2 \alpha^{\prime}} p^{+}, \quad \alpha_{0}^{x}=\frac{v}{\sqrt{2 \alpha^{\prime}}}\left(x^{+}-\frac{a}{\pi v}\right), \quad \alpha_{0}^{-}=\sqrt{2 \alpha^{\prime}} p^{-},
$$

where $\left[x^{ \pm}, p^{\mp}\right]=-i,\left[p^{+}, p^{-}\right]=\left[x^{+}, x^{-}\right]=0$. Because of the Dirichlet condition of $X^{x}$ at $\sigma=0$, the zero modes $x$ and $p_{x}$ do not appear in the mode expansion.2

2 It is well known that the T-dual coordinates satisfying (5.9) are non-commutative at the boundary. However, the original coordinates (5.11) are not non-commutative. 
As in the case of closed string, the commutation relation (5.12) is written as an ordinary relation using the spectral flow

$$
\alpha_{n}=S_{\frac{v}{n}} \alpha_{n}^{(0)}, \quad\left[\alpha_{n}^{(0) \mu} \alpha_{m}^{(0) \nu}\right]=n \delta_{n+m, 0} \eta^{\mu \nu}
$$

In terms of this spectral flowed oscillator, the Virasoro operator of this system is written as 3

$$
L_{0}^{(k)}=-2 \alpha^{\prime} p^{+} p^{-}+\alpha^{\prime}\left(\frac{r}{2 \pi \alpha^{\prime}}\right)^{2}+2 k E+N^{(0)} .
$$

Here $r^{2}$ is defined as

$$
r^{2}=\left(2 \pi k x^{+}-a\right)^{2}+(2 \pi k R-b)^{2}
$$

The $r^{2}$ term in $L_{0}^{(k)}$ has a simple physical interpretation. This term represents the (mass) ${ }^{2}$ of the string stretched between D1-branes at $(x, z)=\left(2 \pi k x^{+}, 2 \pi k R\right)$ and $(x, z)=(a, b)$. Although we are not taking the lightcone gauge, this form of $L_{0}^{(k)}$ suggests that $x^{+}$is the good "time" coordinate to describe this system (see fig. 1). This mass term agrees with the mass of the off-diagonal element around the diagonal configuration $X^{x}=\operatorname{diag}\left(2 \pi k x^{+}, a\right)$ in the low energy Yang-Mills description of this system [25]. It is easy to construct a physical state of the open string with zero mode only

$$
\psi=\exp \left[-i p^{+} x^{-}-i \frac{k^{2}}{6 p^{+}\left(\alpha^{\prime}\right)^{2}}\left(x^{+}-\frac{a}{2 \pi k}\right)^{3}-i \frac{m^{2}}{2 p^{+}} x^{+}\right], \quad m^{2}=\left(\frac{2 \pi k R-b}{2 \pi \alpha^{\prime}}\right)^{2}-\frac{1}{\alpha^{\prime}},
$$

which is the same as the wave-function of the off-diagonal scalar field in the Yang-Mills description [25]. When $p^{+}=0$, only massless states are physical, and they are localized at $x^{+}=a / 2 \pi k$.

One can easily show that the open string 1-loop amplitude

$$
\mathcal{A}_{\text {open }}=\int_{0}^{\infty} \frac{d t}{t} \sum_{k \in \mathbf{Z}} \operatorname{Tr} e^{-2 \pi t\left(L_{0}^{(k)}-\frac{c}{24}\right)}
$$

agrees with the closed string computation (5.5). Here again, the trace over the non-zero modes is independent of $k$.

3 The zero-mode part of our $L_{0}^{(k)}$ is different from the $L_{0}$ of the open string connecting two D2-branes with a null flux on one of the branes [22]. This is consistent since the $X^{x}$-direction is non-compact. We just used the T-duality as a solution generating technique to find the mode expansion in the original D1-brane picture. In appendix B, we discuss the conpactification of the $X^{x}$-direction. 

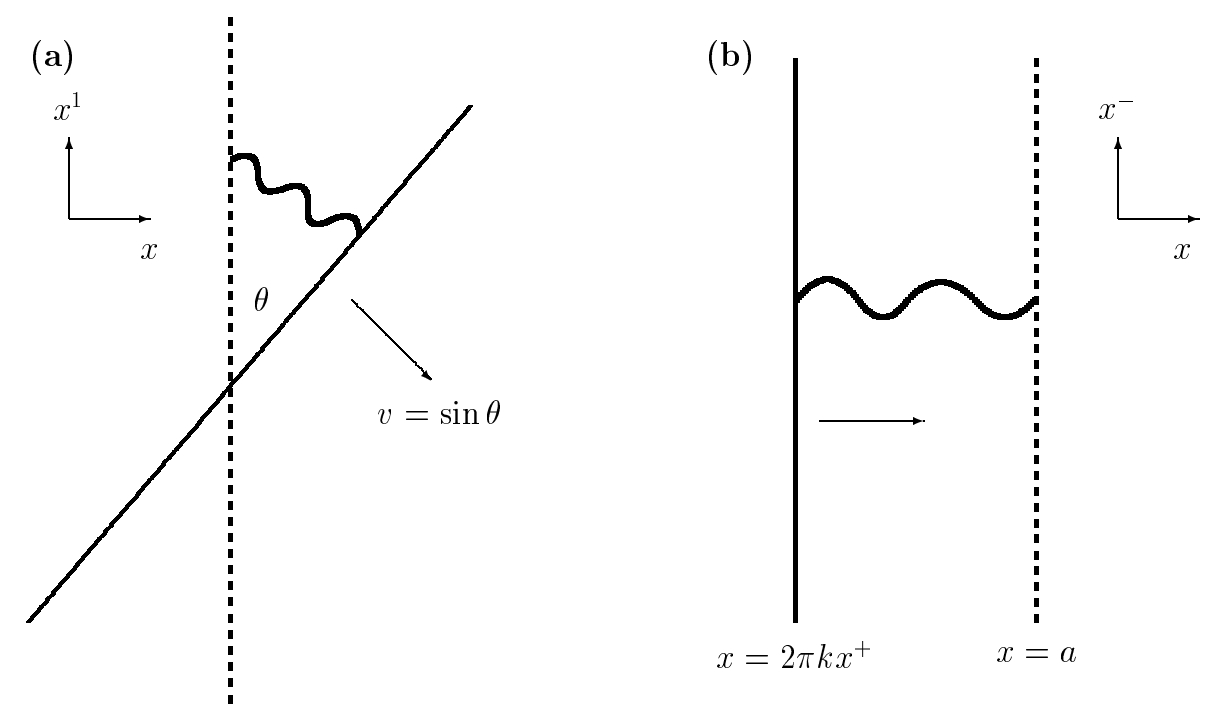

Fig. 1 Two different constant "time" slices of null-scissors: (a) $x^{0}=$ constant: Two branes are intersecting at angle $\theta=\tan ^{-1}(\sqrt{2} \pi k)$. The intersection point moves with the speed of light. (b) $x^{+}=$constant: Two parallel branes collide at $x^{+}=a / 2 \pi k$ and then move apart.

\section{Discussions}

In this paper, we have constructed the boundary states describing D-branes in the null-brane background. It was pointed out that the low energy theory on a brane in this background is described by a noncommutative field theory with a time-dependent noncommutativity [19] (see also [26,22,28]). It is interesting to derive this noncommutativity from the worldsheet viewpoint. One can study the worldvolume theory on the brane by computing a scattering amplitude of open string modes in a similar way as the closed string computation in [8, 16, [17]. For instance, the scattering amplitude of open string tachyons is obtained by a transform of the Veneziano amplitude in stead of the Virasoro-Shapiro amplitude studied in [8, 16, 17]. One can expect that open string amplitudes have a similar behavior as the closed string ones. It is also interesting to study the geometry of the null-brane seen by D-branes. The instantonic brane, or S-brane, would be a good probe, and the noncommutative nature of the transverse coordinates of the brane would have an important role as in [6]. The K-theory and the clarification of stable branes in the null-brane geometry are also interesting.

\section{Acknowledgments}

I would like to thank B. Craps and S. Sethi for useful discussions, and C. Bachas for email correspondence. 


\section{Appendix A. Trace of the Zero-Mode Part}

The trace of the zero-mode part in the twisted sector typically has the form

$$
Z=\operatorname{Tr} e^{-i T\left(\hat{p}^{2}+\lambda \hat{x}\right)}
$$

where $\widehat{x}$ and $\widehat{p}$ are canonically conjugate to each other: $[\widehat{x}, \widehat{p}]=i$. Using the BakerCampbell-Hausdorff formula

$$
\exp X \exp Y=\exp \left(X+Y+\frac{1}{2}[X, Y]+\frac{1}{12}[X-Y,[X, Y]]+\cdots\right),
$$

we can factorize the operator in the trace as

$$
e^{-i T\left(\hat{p}^{2}+\lambda \hat{x}\right)}=e^{-i T\left(\hat{p}^{2}+T \lambda \hat{p}+\frac{1}{3} T^{2} \lambda^{2}\right)} e^{-i T \lambda \hat{x}} .
$$

Here, the dots in the $\mathrm{BCH}$ formula vanishes since $\left[\widehat{x},\left[\widehat{x}, \widehat{p}^{2}\right]\right]=-2$ is a c-number. Then we can evaluate the trace in the plane-wave basis

$$
\begin{aligned}
Z & =\int d x d p\left\langle p\left|e^{-i T\left(\hat{p}^{2}+T \lambda \hat{p}+\frac{1}{3} T^{2} \lambda^{2}\right)}\right| x\right\rangle\left\langle x\left|e^{-i T \lambda \hat{x}}\right| p\right\rangle=\int \frac{d x d p}{2 \pi} e^{-i T\left(p^{2}+T \lambda p+\frac{1}{3} T^{2} \lambda^{2}\right)} e^{-i T \lambda x} \\
& =\int d p e^{-i T p^{2}} \delta(T \lambda)=\int \frac{d x d p}{2 \pi} e^{-i T\left(p^{2}+\lambda x\right)}
\end{aligned}
$$

Namely, the trace is equal to the classical integral over the phase space. Notice that it is important to use the Lorentzian proper time $T \in \mathbf{R}$.

\section{Appendix B. T-dual of the Null-Scissors}

In this appendix, we consider the relation of the null-scissors and its T-dual configuration. The T-dual of the null-scissors is the two D2-branes with a constant null flux $F^{-x}$ on one of them. The open string stretched between these two D2-branes satisfies the boundary conditions (5.9), and the mode expansion is given by (5.10). The commutators of the oscillators are the same as the original D1-brane picture (5.12), the commutator of the zero modes are [22]

$$
\left[x^{\mu}, \alpha_{0}^{\nu}\right]=i \sqrt{2 \alpha^{\prime}} \eta^{\mu \nu}, \quad\left[\alpha_{0}^{x}, \alpha_{0}^{-}\right]=-i v .
$$

These zero-modes $\alpha_{0}^{\mu}$ can be written in terms of the canonical variables $x^{\mu}$ and $p^{\mu}$, obeying $\left[x^{\mu}, p^{\nu}\right]=i \eta^{\mu \nu},\left[x^{\mu}, x^{\nu}\right]=\left[p^{\mu}, p^{\nu}\right]=0$, as $\alpha_{0}^{+}=\sqrt{2 \alpha^{\prime}} p^{+}$and

$$
\alpha_{0}^{x}=\sqrt{2 \alpha^{\prime}} p^{x}+f x^{+}+g x, \quad \alpha_{0}^{-}=\sqrt{2 \alpha^{\prime}} p^{-}+f^{\prime} x+g^{\prime} x^{+} .
$$


Here $g$ and $g^{\prime}$ are arbitrary constants and $f$ and $f^{\prime}$ are related by

$$
f+f^{\prime}=\frac{v}{\sqrt{2 \alpha^{\prime}}}
$$

The value of these parameters $f, f^{\prime}, g, g^{\prime}$ can be changed by the canonical transformation of $x^{\mu}$ and $p^{\mu}$.

To see the relation of this T-dual picture with the original D1-brane picture, let us compactify the coordinate $Y^{x}$ on a circle of radius $L: Y^{x} \sim Y^{x}+2 \pi L$. Then the momentum $p^{x}$ in this direction is quantized as $p^{x}=m / L, m \in \mathbf{Z}$. By performing the T-duality along the $Y^{x}$-direction, the mode expansion of the original coordinate reads

$$
\begin{aligned}
& X^{+}=x^{+}+\sqrt{2 \alpha^{\prime}} t \alpha_{0}^{+}+\text {(oscillators) } \\
& X^{x}=x+\sqrt{2 \alpha^{\prime}}\left(\sigma \alpha_{0}^{x}+v \sigma t \alpha_{0}^{+}\right)+\text {(oscillators). }
\end{aligned}
$$

At the boundary of the open string, these coordinates satisfy

$$
\left.X^{x}\right|_{\sigma=0}=x, \quad X^{x}-\left.\pi v X^{+}\right|_{\sigma=\pi}=x-\pi v x^{+}+\sqrt{2 \alpha^{\prime}} \pi \alpha_{0}^{x} .
$$

Using the freedom of the choice of parameters $f, f^{\prime}, g, g^{\prime}$, we take the following form of the zero-modes:

$$
\alpha_{0}^{x}=\sqrt{2 \alpha^{\prime}} p^{x}+\frac{1}{\pi \sqrt{2 \alpha^{\prime}}}\left(\pi v x^{+}-x\right), \quad \alpha_{0}^{-}=\sqrt{2 \alpha^{\prime}} p^{-} .
$$

Then $X^{x}-\pi v X^{+}$satisfies the Dirichlet boundary condition at the boundary $\sigma=\pi$

$$
X^{x}-\left.\pi v X^{+}\right|_{\sigma=\pi}=2 \pi \alpha^{\prime} p^{x}=2 \pi m \widetilde{L}
$$

where $\widetilde{L}=\alpha^{\prime} / L$ is the radius of the circle in the $X^{x}$-direction. In other words, the "moving D1-brane" of the null-scissors have infinitely many image branes periodically sitting along the $X^{x}$-direction. Now let us take the decompactification limit $\widetilde{L} \rightarrow \infty$. In the T-dual picture $L \rightarrow 0$, the momentum modes $p^{x}=m / L$ with $m \neq 0$ become infinitely massive and hence decouple. In the original picture, the images of the "moving brane" sitting at (B.7) with $m \neq 0$ go to infinity and decouple. Therefore, we can set $p^{x}=0$ in the decompactification limit, and we can also set the zero-mode $x$ of $X^{x}$ to be a constant a. Thus we recover the mode expansion (5.11)-(5.13) in the D1-brane picture with the non-compact $X^{x}$ coordinate. Note that the coordinates $X^{\mu}$ (5.11) satisfy the canonical commutation relation without including the contribution of the zero-modes $x$ and $p^{x}$ 国

\footnotetext{
4 The zero-modes with $f^{\prime}=v / \sqrt{2 \alpha^{\prime}}$ and $f=g=g^{\prime}=0$ are considered in [22].

5 Recall that a boson $\phi=\phi_{0}+\sqrt{2 \alpha^{\prime}} \sigma \alpha_{0}+\sqrt{2 \alpha^{\prime}} \sum_{n \neq 0} \alpha_{n} / n e^{-i n t} \sin n \sigma$ obeying the Dirichlet boundary condition satisfies the commutation relation $\left[\phi(\sigma), \partial_{t} \phi\left(\sigma^{\prime}\right)\right]=2 \pi i \alpha^{\prime} \delta\left(\sigma, \sigma^{\prime}\right)$ with the $\delta$-function $\delta\left(\sigma, \sigma^{\prime}\right)=1 / \pi \sum_{n \neq 0} \sin n \sigma \sin n \sigma^{\prime}$. Since the commutator $\left[\phi_{0}, \alpha_{0}\right]$ does not appear in this relation, we can consistently set $\phi_{0}$ and $\alpha_{0}$ to be c-numbers which specify the position of D-branes.
} 


\section{References}

[1] G. T. Horowitz and A. R. Steif, "Singular String Solutions With Nonsingular Initial Data," Phys. Lett. B 258, 91 (1991).

[2] J. Khoury, B. A. Ovrut, N. Seiberg, P. J. Steinhardt and N. Turok, "From big crunch to big bang," Phys. Rev. D 65, 086007 (2002) hep-th/0108187.

[3] J. Figueroa-O'Farrill and J. Simon, "Generalized supersymmetric fluxbranes," JHEP 0112, 011 (2001) hep-th/0110170.

[4] V. Balasubramanian, S. F. Hassan, E. Keski-Vakkuri and A. Naqvi, "A space-time orbifold: A toy model for a cosmological singularity," hep-th/0202187.

[5] L. Cornalba and M. S. Costa, "A New Cosmological Scenario in String Theory," Phys. Rev. D 66, 066001 (2002) hep-th/0203031.

[6] N. A. Nekrasov, "Milne universe, tachyons, and quantum group," hep-th/0203112.

[7] J. Simon, "The geometry of null rotation identifications," JHEP 0206, 001 (2002) hep-th/0203201.

[8] H. Liu, G. Moore and N. Seiberg, "Strings in a time-dependent orbifold," JHEP 0206, 045 (2002) hep-th/0204168.

[9] O. Aharony, M. Fabinger, G. T. Horowitz and E. Silverstein, "Clean time-dependent string backgrounds from bubble baths," JHEP 0207, 007 (2002) hep-th/0204158.

[10] S. Elitzur, A. Giveon, D. Kutasov and E. Rabinovici, "From big bang to big crunch and beyond," JHEP 0206, 017 (2002) [hep-th/0204189].

[11] L. Cornalba, M. S. Costa and C. Kounnas, "A resolution of the cosmological singularity with orientifolds," Nucl. Phys. B 637, 378 (2002) hep-th/0204261.

[12] B. Craps, D. Kutasov and G. Rajesh, "String propagation in the presence of cosmological singularities," JHEP 0206, 053 (2002) [hep-th/0205101].

[13] A. Lawrence, "On the instability of 3D null singularities," hep-th/0205288.

[14] V. Balasubramanian and S. F. Ross, "The dual of nothing," Phys. Rev. D 66, 086002 (2002) hep-th/0205290.

[15] A. Maloney, E. Silverstein and A. Strominger, "De Sitter space in noncritical string theory," hep-th/0205316.

[16] H. Liu, G. Moore and N. Seiberg, "Strings in time-dependent orbifolds," JHEP 0210, 031 (2002) hhep-th/0206182.

[17] M. Fabinger and J. McGreevy, "On smooth time-dependent orbifolds and null singularities," [hep-th/0206196].

[18] G. T. Horowitz and J. Polchinski, "Instability of spacelike and null orbifold singularities," hep-th/0206228.

[19] A. Hashimoto and S. Sethi, "Holography and string dynamics in time-dependent backgrounds," hep-th/0208126. 
[20] J. Simon, "Null orbifolds in AdS, time dependence and holography," JHEP 0210, 036 (2002) hep-th/0208165.

[21] E. Dudas, J. Mourad and C. Timirgaziu, "Time and space dependent backgrounds from nonsupersymmetric strings," hep-th/0209176.

[22] C. Bachas and C. Hull, "Null brane intersections," hep-th/0210269.

[23] J. Figueroa-O'Farrill and J. Simon, "Supersymmetric Kaluza-Klein reductions of M2 and M5 branes," hep-th/0208107.

[24] J. Figueroa-O'Farrill and J. Simon, "Supersymmetric Kaluza-Klein reductions of Mwaves and MKK-monopoles," hep-th/0208108.

[25] R. C. Myers and D. J. Winters, "From D - anti-D pairs to branes in motion," hepth/0211042.

[26] M. Alishahiha and S. Parvizi, "Branes in time-dependent backgrounds and AdS/CFT correspondence," JHEP 0210, 047 (2002) [hep-th/0208187.

[27] L. Dolan and C. R. Nappi, "Noncommutativity in a time-dependent background," hep-th/0210030.

[28] R. G. Cai, J. X. Lu and N. Ohta, "NCOS and D-branes in time-dependent backgrounds," hep-th/0210206. 